\title{
Sinais vitais e expressão facial de pacientes em estado de coma*
}

\author{
Vital signs and facial expression of patients in coma
}

Signos vitales y expresión facial de pacientes en estado de coma

\section{Ana Cláudia Giesbrecht Puggina', Maria Júlia Paes da Silva' \\ 'Universidade de São Paulo. Escola de Enfermagem, Departamento de Enfermagem Médico-Cirúrgica. São Paulo, SP}

Submissão: $23 / 07 / 2008$

Aprovação: 15/04/2009

\section{RESUMO}

O objetivo foi verificar a influência da música e mensagem oral sobre os Sinais Vitais e Expressão Facial dos pacientes em coma fisiológico ou induzido. Realizou-se um Ensaio Clínico Controlado e Randomizado. A amostra consistiu-se de 30 pacientes de Unidade de Terapia Intensiva, Que foram divididos em 2 grupos: Grupo Controle (sem estímulos auditivos) e Grupo Experimental (com estímulos auditivos). Os pacientes foram submetidos a 3 sessões, em dias consecutivos. Encontraram-se alterações estatisticamente significativas nos sinais vitais (saturação de $\mathrm{O}_{2}$ - sessão I; saturação de $\mathrm{O}_{2}$ - sessão 3; freeüência respiratória - sessão 3) durante a mensagem e na expressão facial, sessão I, durante a música e a mensagem. Aparentemente a mensagem foi um estímulo mais forte do Que a música em relação à capacidade de produzir respostas fisiológicas sugestivas de audição.

Descritores: Coma; Música; Unidades de terapia intensiva.

\begin{abstract}
The objective was to check music and voice message influence on the Vital Signals and Facial Expressions of patients in physiological or induced comas. A Randomized Controlled Clinical Trial was done. The sample was consisted of 30 patients from Intensive Care Unit, being divided in 2 groups: Group Control (without auditory stimuli) and Experimental Group (with auditory stimuli). The patients were submitted to 3 sessions for consecutive days. Significant statistical alterations of the vital signs were noted (oxygen saturation - session 1; oxygen saturation - session 3; respiratory freeuency - session 3) during the message playback and with facial expression, session I, during both music and message. Apparently, the voice message is a stronger stimulus than the music in relation to the capacity of producing suggestive physiological auditory responses.
\end{abstract}

Descriptors: Coma; Music; Intensive care units.

\section{RESUMEN}

El objetivo era verificar la influencia de la música y del mensaje verbal en los Señales Vitales y la Expresión Facial de los pacientes en coma fisiológico o inducido. Un Ensayo Clínico Controlado y Randomizado fue echo. La muestra fue consistida en 30 pacientes de Unidad de terapia Intensiva, Que fueran divididos en 2 grupos: Grupo Control (sin estímulos auditivos) y Grupo Experimental (con los estímulos auditivos). Los pacientes fueran sometidos a las 3 sesiones, en días consecutivos. Los cambios estadísticamente significativos en las Señales Vitales fueran encuentrados (saturación del oxigeno - sesión 1; saturación del oxigeno - sesión 3; frecuencia respiratoria - sesión 3) durante el mensaje y en la Expresión Facial, sesión I, durante música y el mensaje. Aparentemente el mensaje era uno estimulo más fuerte de qué la música en lo Que refiere a la capacidad de producir respuestas fisiológicas de audición.

Descriptores: Coma; Musica; Unidades de Terapia Intensiva.

* Dados parciais extraído da dissertação "O uso da música e de estímulos vocais em pacientes em estado de coma: relação entre estímulo auditivo, sinais vitais, expressão facial e Escalas de Glasgow e Ramsay", EEUSP, 2006. 


\section{INTRODUÇÃO}

O coma (do grego kôma = sono profundo) pode ser definido como estado de perda total ou parcial da consciência, da motricidade voluntária e da sensibilidade, geralmente devido a lesões cerebrais, intoxicações, problemas metabólicos e endócrinos, no Qual, dependendo da gravidade, as funções vitais são mantidas em maior ou menor grau(I). Quando fisiológico, o estado de coma pode ser mensurado através da Escala de Coma de Glasgow (ECGl) e Quando farmacológico pela Escala de Sedação de Ramsay (ESR).

A percepção auditiva dos pacientes em coma, tanto fisiológico como induzido, em relação à comunicação verbal ao seu redor sempre foi muito Questionada e, até hoje, mesmo com os avanços da Medicina e da neurociência, ainda não temos uma resposta precisa do que acontece na mente desses pacientes durante a experiência de estar em coma.

A audição parece ser o último sentido Que é perdido, e tal afirmação pode ser sustentada através dos relatos de pessoas Que retornaram desse estado. A maioria relata dados sensoriais auditivos como sons, palavras, frases, vozes familiares etc. Por isso, é extremamente importante o ambiente sonoro em Que o paciente está inserido, bem como as conversas paralelas ao lado do leito e a própria comunicação com o paciente, principalmente antes da realização de QualQuer procedimento.

Relatos como o de $\operatorname{Scotti}^{(2)}$ e do italiano Salvatore Crisafulli ${ }^{(3)}$, largamente divulgados pela mídia, são histórias Que sugerem a existência de audição nesses pacientes durante o estado de coma e, talvez, também a existência de estados de consciência diferentes dos Que conhecemos.

"Eu estive Quase dois meses em coma, e lembro-me muita coisa, visitas de amigos e familiares, palavras encorajadoras e também algumas atitudes frias e até desleixadas da equipe de enfermagem e médica. Não é nada agradável ouvir, estando em coma, algum médico dizendo a um parente seu Que você irá morrer. Eu ouvi, mas naquele momento, ouvir era o máximo que eu fazia..."(2).

"Os médicos diziam Que eu não estava consciente, mas eu entendia tudo e gritava desesperado. Mas eu não podia falar, não podia me mover, não podia fazer nada para fazê-los entender Que eu os ouvia. Com isso, chorava..."(3).

Como existem muitas duvidas em relação ao Que ocorre com o paciente em coma precisamos aprender outros meios de percebêlos. Manifestações ou correlatos fisiológicos das emoções são respostas autonômicas, comandadas pelo Sistema Nervoso Autônomo (SNA), frente a um estímulo. Aumento da freeüência cardíaca, respiratória e rubor da face são exemplos de manifestações fisiológicas de uma emoção positiva. Uma emoção negativa também desencadeia aumento da freeüência cardíaca e respiratória, mas ao contrario da anterior, o individuo empalidece e sua frio. As emoções também podem provocar manifestações comportamentais, isto é, respostas motoras e essas podem ser estereotipadas (reflexas ou involuntárias) ou até bastante complexas envolvendo ações voluntárias ${ }^{(4)}$.

Os sinais vitais podem fornecer informações relevantes sobre o estado físico e emocional dos pacientes, sendo importante conhecer como estes são controlados e Quais são as suas relações intrínsecas.

As alterações na freQüência cardíaca são efetuadas por controles reflexos mediados pelo SNA, incluindo suas divisões simpática e parassimpática. Os impulsos parassimpáticos Que chegam até o coração por meio do nervo vago, podem lentificar a freoüência cardíaca, en\&uanto os impulsos parassimpáticos a aumentam. Esses efeitos sobre a freqüência cardíaca resultam da ação sobre o nódulo sinoatrial, Quer para diminuir, Quer para aumentar sua freeüência inerente. $\mathrm{O}$ equilíbrio entre esses dois sistemas de controle reflexo normalmente determina a freqüência cardíaca, mas essa também é estimulada por um nível aumentado de catecolaminas circulantes (secretadas pela supra-renal) e pelo excesso de hormônio tireóideo, 0 Qual provoca um efeito semelhante à catecolamina ${ }^{(5)}$.

Além disso, a freqüência cardíaca também é afetada pelo SNC. Os barorreceptores, células nervosas especializadas localizadas no arco aortico e nas artérias carótidas, são sensíveis às alterações na pressão arterial. Durante as elevações na pressão arterial, essas células aumentam suas freeüências de descarga, transmitindo os impulsos para a medula. Isso inicia a atividade parassimpática e inibe a resposta Simpática, diminuindo a freeüência cardíaca e a pressão arterial, o oposto também é verdadeiro(5).

Os mecanismos regulares da pressão arterial incluem o córtex cerebral, o hipotálamo, os centros vasomotores, o SNA, as suprarenais, os rins, os barorreceptores e algumas vias nervosas especiais, como o nervo de Cyon e o de Hering. O sistema humoral, a cargo dos rins e das suprar-renais, é mediado por várias substancias - renina, aldosterona, angiotensina, prostaglandinas, vasopressina, desoxicorticosterona e glicocorticóides ${ }^{(6)}$.

Em relação ao sistema respiratório, o simpático provoca broncodilatação e o parassimpático constrição brôn@uica. Os músculos respiratórios são estriados esqueléticos, estão sob comando de motoneurônios medulares e sob controle das vias descendentes. Por isso, os parâmetros dos movimentos são determinados por regióes do tronco encefálico, a partir de informações provenientes de Quimiorreceptores aórticos e carotídeos, bem como mecanorreceptores situados nas paredes das vias aéreas ${ }^{(4)}$.

A termorregulação funciona com um ponto de ajuste hipotalâmico Que fica em torno de $37^{\circ} \mathrm{C}$ na maioria dos casos. Os termorreceptores periféricos e centrais constituem o sistema de retroação, o hipotálamo é o integrador e o controlador é múltiplo, formado pelo SNA, sistema endócrino e sistema neuromuscular ${ }^{(4)}$.

O hipotálamo anterior ativa os controladores sub-reguladores, isto é, capazes de diminuir o tônus vascular simpático periférico e de provocar a sudorese e o aumento da fręüência e amplitude respiratórias, garantindo a dissipação de calor corporal excessivo. O hipotálamo posterior, ao contrario, ativa os controladores supra-reguladores, ou seja, ąueles capazes de provocar a estimulaçãa da inervação simpática dos vasos cutâneos e os tremores musculares involuntários, provocando a conservação e a geração de calor corporal( ${ }^{(4)}$.

Alguns trabalhos ${ }^{(7-10)}$ Que se propuseram estudar a percepção dos pacientes em estado de coma utilizaram sinais vitais ou respostas comportamentais como indicadores de resultado.

Aldridge et $\mathrm{a}^{(7)}$ aplicaram musicoterapia através de uma canção vocalizada por uma terapeuta em 5 pacientes e avaliaram freqüência respiratória, fręüência cardíaca e alterações comportamentais. Foram encontradas uma variedade de reações: mudança na respiração (ela se tornava mais lenta e mais profunda), movimentos de motor fino, movimentos de fechar as mãos e virar a cabeça, abrir os olhos para a recuperação da consciência. Quando a 
terapeuta começava a cantar havia uma diminuição da freQüência cardíaca. Então, esta se elevava rapidamente e se sustentava nesse nível elevado até o final do contato. Isto pode indicar uma tentativa de orientação e reconhecimento do estímulo.

Sisson ${ }^{(8)}$ utilizou como estímulo duas músicas populares préselecionadas e avaliou através de Eletroencefalograma (EEG) o efeito da entrada sensorial específica na atividade cortical de 5 pacientes em estado de coma. Os resultados indicaram Que os pacientes em coma puderam responder ao estímulo auditivo. As respostas foram variadas nesta peQuena amostra e, portanto, inconclusivas do ponto de vista científico, mas algum tipo de resposta ocorreu. Dois pacientes demonstraram uma resposta no EEG e os outros três responderam pela abertura ocular ou movimentação de extremidade.

Iones et $\mathrm{al}^{(9)}$ aplicaram 4 tipos de estímulos auditivos (vozes de familiares e amigos, música clássica, música popular de preferência e sons da natureza) em I paciente jovem (16 anos), do sexo masculino, vítima de um acidente de trânsito, comatoso, 42 dias pós-trauma. Foram avaliadas a freeüência respiratória, a fręüência cardíaca e a resistência cutânea do paciente frente aos diferentes estímulos. O estímulo de vozes familiares e amigos, comparado aos outros estímulos auditivos, foi o Que produziu um maior aumento no pulso e na freQüência respiratória em relação às medidas basais, bem como o Que resultou no maior número de movimentos corporais.

Walker et $\mathrm{al}^{(10)}$ avaliaram o efeito da intervenção através de vozes familiares em 10 pacientes comatosos com trauma craniano. Foram observados os parâmetros fisiológicos (pressão intracraniana, pressão arterial, pulso, freQüência respiratória, pressão arterial média e saturação de oxigênio). Os pacientes possuíam pontuação na ECGl menor ou igual a 10. A análise dos dados revelou apenas uma variabilidade nas medidas fisiológicas; não foram encontradas diferenças estatisticamente significantes entre a média das medidas antes, durante e após a mensagem. Entretanto, o pulso foi uma medida Que teve uma maior variabilidade em relação aos outros parâmetros fisiológicos.

Esses estudos ${ }^{(7-10)}$, de uma maneira ou de outra, apontam para a existência de certa responsividade. Porém, mais estudos precisam ser feitos para determinar outros métodos capazes de medir essas respostas de uma maneira mais conclusiva, pois o universo de estudos nessa linha de pesquisa ainda é muito peeueno ${ }^{(1,12)}$.

\section{OBJETIVO}

Verificar a influência da música e mensagem oral sobre os sinais vitais e expressão facial dos pacientes em coma fisiológico ou induzido.

\section{MÉTODO}

\section{Tipo de Estudo}

Realizou-se um Ensaio Clínico Controlado e Randomizado(13) tendo como intervenção uma música de preferência do paciente e uma mensagem oral (variáveis independentes) e como indicadores de resultados as alterações nos sinais vitais e na expressão facial (variáveis dependentes).

\section{Amostra}

A amostra constituiu-se de 30 pacientes internados em UTIs de um hospital escola da cidade de São Paulo no período de março a setembro de 2005 que atendiam aos seguintes critérios de inclusão: ( I) estar em coma; (2) ser avaliado segundo uma das Escalas e ter a pontuação delimitada para o estudo (Escore entre 3 a 8 na ECGl se o coma fisiológico e R5 ou R6 na ESR se o coma induzido); (3) ter função auditiva preservada segundo familiares, ou seja, excluíramse pessoas surdas ou com acuidade auditiva previamente diminuída; (4) ter concordância por escrito da família ou responsável para a participação do paciente na pesQuisa.

\section{Intervenção e Coleta dos Dados}

Iniciou-se a coleta de dados após a aprovação do Comitê de Ética e assinatura do Termo de Consentimento Livre e Esclarecido pelo familiar.

Os pacientes foram alocados aleatoriamente em dois grupos, Experimental e Controle, sendo Que apenas o Grupo Experimental recebeu a intervenção, música e mensagem.

A mensagem oral foi utilizada com dois objetivos básicos: expor o paciente a um estímulo de linguagem e focalizar a atenção do paciente. Para sua elaboração utilizamos alguns critérios de padronização: (1) elaboração da mensagem por um familiar significativo para o paciente, pois nem sempre a pessoa Que o estava acompanhando naQuele momento tinha um forte vínculo; (2) duração de, no máximo, 3 minutos; (3) dizer inicialmente e no fim da mensagem Quem estava falando; (4) falar 3 vezes, no mínimo, o nome do paciente no decorrer da mensagem; (5) situar o paciente espacialmente: onde o paciente estava e o que estava acontecendo com ele; (6) dizer uma mensagem de carinho e com uma perspectiva otimista; contando algo de sua vida familiar.

A música utilizada no presente estudo foi selecionada pelo familiar, considerando a preferência do paciente, e visava oferecer um estímulo Que tivesse um vínculo afetivo com o paciente, além de garantir-lhe conforto.

Para cada paciente confeccionou-se 2 Compact Disc (CD). Um com os estímulos sonoros (música de preferência e mensagem) e outro com o mesmo tempo de gravação do $\mathrm{CD}$ anterior, mas sem estímulos sonoros. O CD sem estímulos foi obtido através da gravação de silêncio realizada com o gravador de voz digital e posteriormente gravada no $\mathrm{CD}$. Como o tempo de gravação variou de acordo com a música escolhida e a mensagem, não houve na pesQuisa um tempo padrão de duração dos CDs.

A partir disso, a alocação randomizada dos pacientes nos dois grupos foi feita. Para o Grupo Experimental tocou-se o CD com os estímulos sonoros e para o Grupo Controle o CD sem os estímulos sonoros. Colocou-se fones de ouvidos nos pacientes durante as sessões.

A orientadora foi responsável pela distribuição aleatória dos grupos, sem Que a pesquisadora soubesse Qual o CD escolhido (unicego para o pesquisador), evitando assim, com esse mascaramento, tendenciosidade de interpretação na coleta de dados. $\mathrm{O}$ volume dos estímulos sonoros foi entre 60 e 70 decibéis.

A avaliação segundo a ECGl e a ESR foi realizada sempre no início de cada sessão e antes de QualQuer estímulo.

Os pacientes foram submetidos a 3 sessões, sendo realizada I por dia em 3 dias consecutivos. Dias consecutivos possibilitaram uma chance maior dos dados serem coletados com o paciente no mesmo estado clínico. O número de sessões foi importante para 
avaliar se os estímulos influenciaram no estado mental do paciente.

Elaborou-se um instrumento para coleta dos dados. No instrumento, em cada uma das sessões, foram anotados os sinais vitais e a expressão facial dos pacientes como parâmetros para avaliar suas reações fisiológicas frente aos estímulos. Os sinais vitais foram avaliados após 60 segundos de cada estímulo e anotados numa seQüência pré-estabelecida: pulso, temperatura axilar, pressão arterial, saturação de oxigênio $\left(\mathrm{O}_{2}\right)$ e freQüência respiratória. Essa seqüência foi estabelecida apenas com o critério de praticidade e padronização.

Todos os pacientes incluídos na pesquisa estavam monitorizados com monitor hemodinâmico. As medidas de pulso e saturação de $\mathrm{O}_{2}$ foram obtidas através do oxímetro capilar conectado ao monitor. A pressão arterial foi obtida através da solicitação de medida de pressão não-invasiva ao monitor. Em relação à temperatura axilar, foram usadas duas maneiras para a obtenção dos dados: medida de temperatura axilar contínua conectada ao monitor e medida através de termômetro manual de coluna de mercúrio. A freqüência respiratória do paciente foi obtida através do ventilador mecânico.

A expressão facial foi avaliada imediatamente antes das sessões e durante elas com a ajuda de filmagem, para Que nenhum dado fosse perdido durante a coleta de dados. As filmagens foram realizadas por câmera digital e transferidas para o computador para posterior visualização.

\section{Tratamento dos Dados}

Os dados foram armazenados no programa Excel for Windows Explorer ${ }^{\circledR}$ e as análises foram feitas no programa STATA $®$ visando comparar os dois grupos. Para a comparação das variáveis utilizamos o Teste Exato de Fisher, Teste de Mann-Whitney. A probabilidade de ocorrência estatística $(p)$ dos testes considerou a diferença: (1) significativa se $p$ inferior a 0,05 ou 0,10 ; (2) tendência de significância se $p$ entre 0,10 e 0,20 ; (3) não significativa se o $p$ superior a 0,20 .

\section{RESULTADOS E DISCUSSÃO}

\section{Descrição da Amostra}

Comparando os dois grupos, Experimental e Controle, segundo o Teste Exato de Fisher, em relação à faixa etária $(p=1,00)$, causas do rebaixamento do nível de consciência $(p=0,46)$ e dias de internação $(p=0,81)$ estes não diferem estatisticamente entre si, ou seja, os grupos podem ser considerados homogêneos e, portanto, comparados.

O Grupo Experimental tinha em média 44,7 anos ( \pm 1 8,7 desviopadrão) e 12 dias de internação. O Grupo Controle tinha 47,7 anos em média ( $\pm 21,8$ desvio-padrão) e 22,2 dias de internação. Quanto às causas do rebaixamento do nível de consciência de uma forma geral, sedação (11 pacientes, 36\%) e TCE (11 pacientes, $36 \%)$ foram as principais causas.

Pierin et $\mathrm{al}^{(14)}$, ao caracterizarem os pacientes de duas UTIs gerais, observaram Que as faixas etárias predominantes corresponderam à de 61 a 80 anos $(50,5 \%)$, seguida de 41 a 60 anos (33\%). As UTIs costumam ter um perfil de pacientes com faixa etária mais elevada; porém, como grande parte dos pacientes nesse estudo estavam em uma UTI de Trauma, a média de idade encontrada foi menor por conter um número considerável de pacientes mais jovens, população com maior incidência a traumas graves, por ser mais exposta.

\section{As Músicas}

As músicas escolhidas foram muito variadas, mesmo poreue, no Que diz respeito à faixa etária, nível social e até origem etnológica, os pacientes eram muito diferentes e, na maioria das vezes, essas músicas estavam relacionadas com um momento significativo na vida do paciente, personalidade, experiências positivas etc.

McCaffrey e Locsin ${ }^{(15)}$ consideram a preferência musical um dos princípios, além de outros, Que devem ser considerados para tornar a música uma intervenção de Enfermagem integral. A preferência musical leva em consideração experiências pessoais anteriores como a escuta da música, idade, cultura, estado de espírito, gênero e atitude. É previsto Que exista uma correlação positiva entre o grau de significância Que a música tem na vida pessoal antes do início da doença e a efetividade da música como intervenção durante essa enfermidade.

\section{As Mensagens}

Todas as mensagens, de uma forma ou de outra, demonstraram saudade e carinho pelos pacientes. Na maioria delas, 27 das 30 mensagens (90\%), independente da religião dos familiares houve uma forte expressão de religiosidade; palavras como Deus, Jesus e oração estiveram muito presentes.

Um estudo ${ }^{(16)}$ levantou os sentimentos dos pacientes internados em UTI em Que a religiosidade envolvida durante a internação foi evidenciada como uma maneira de superar as dificuldades, o Que pode ser facilmente transferida aos familiares Que também passam pela experiência, mas sob outra perspectiva, não menos dolorosa. Segundo o estudo ${ }^{(16)}$, a religiosidade é utilizada pelos pacientes como forma de se sentirem seguros dentro de um ambiente desconhecido, trazendo para perto de si a crença e a proteção por eles perdida àquele em Quem confia. Aquele ser enaltecido é, afinal, muitas vezes, o único ponto de apoio.

A vontade de Que o ente Querido volte logo para casa foi mencionada por 20 dos 30 (66,7\%) familiares. E outros $16(53,3 \%)$ familiares referiram durante a mensagem expressões Que denotam um desejo Que o paciente não se preocupe com os problemas externos e fieue traneüilo durante a hospitalização.

\section{Os Sinais Vitais}

Comparamos estatisticamente os grupos apenas em relação às diferenças entre os valores antes e durante cada um dos estímulos (ou silêncio no Controle) nas 3 sessões. Apesar das médias dos sinais vitais do Grupo Experimental durante a música e a mensagem terem se alterado na grande maioria das vezes em relação à média basal, Quando comparadas com o Grupo Controle, diferenças estatisticamente significantes e tendências de significância foram encontradas em apenas alguns momentos (Tabela I).

Comparando os grupos, encontramos alterações estatisticamente significativas nos seguintes sinais vitais: saturação de $\mathrm{O}_{2}-$ sessão I; saturação de $\mathrm{O}_{2}$ - sessão 3; freeüência respiratória - sessão 3 , todas durante a mensagem.

As tendências de significância foram nas seguintes variáveis: temperatura - sessão 3 durante a mensagem; pressão arterial sistólica - sessão 3 durante a música; pressão arterial diastólica sessão 3 durante a mensagem; freeüência respiratória - sessão 2 
Tabela 1. Aproximação Normal (Z) do Teste Mann-Whitney e valor da probabilidade de ocorrência estatística (p) para a diferença dos sinais vitais entre os grupos, Controle e Experimental, durante as 3 sessões - São Paulo, 2006.

\begin{tabular}{|c|c|c|c|c|}
\hline Variáveis comparadas & Sessão & Mensurações & $Z$ & $P$ \\
\hline \multirow{6}{*}{$\begin{array}{l}\text { PULSO } \\
\text { (bpm) }\end{array}$} & 1 & Música & $-0,23$ & 0,82 \\
\hline & & Mensagem & $-0,08$ & 0,93 \\
\hline & 2 & Música & $-0,59$ & 0,55 \\
\hline & & Mensagem & $-0,25$ & 0,80 \\
\hline & 3 & Música & $-0,52$ & 0,60 \\
\hline & & Mensagem & 0,52 & 0,60 \\
\hline \multirow{6}{*}{$\begin{array}{l}\text { TEMPERATURA } \\
\left({ }^{\circ} \mathrm{C}\right)\end{array}$} & 1 & Música & $-0,07$ & 0,94 \\
\hline & & Mensagem & $-0,23$ & 0,82 \\
\hline & 2 & Música & 0,90 & 0,37 \\
\hline & & Mensagem & $-0,73$ & 0,47 \\
\hline & 3 & Música & $-0,38$ & 0,70 \\
\hline & & Mensagem & 1,28 & 0,20 \\
\hline \multirow{6}{*}{$\begin{array}{l}\text { PRESSÃO ARTERIAL SISTÓLICA } \\
(\mathrm{mmHg})\end{array}$} & 1 & Música & 1,15 & 0,25 \\
\hline & & Mensagem & 0,84 & 0,40 \\
\hline & 2 & Música & $-0,02$ & 0,98 \\
\hline & & Mensagem & $-0,38$ & 0,71 \\
\hline & 3 & Música & 1,43 & 0,15 \\
\hline & & Mensagem & $-1,17$ & 0,24 \\
\hline \multirow{6}{*}{$\begin{array}{l}\text { PRESSÃO ARTERIAL DIASTÓLICA } \\
(\mathrm{mmHg})\end{array}$} & 1 & Música & 0,65 & 0,51 \\
\hline & & Mensagem & 0,06 & 0,95 \\
\hline & 2 & Música & $-0,36$ & 0,72 \\
\hline & & Mensagem & $-1,01$ & 0,31 \\
\hline & 3 & Música & 1,09 & 0,27 \\
\hline & & Mensagem & $-1,56$ & 0,12 \\
\hline \multirow{6}{*}{$\begin{array}{l}\text { SATURAÇÃO DE O2 } \\
\text { (\%) }\end{array}$} & 1 & Música & 1,05 & 0,29 \\
\hline & & Mensagem & 2,09 & 0,04 \\
\hline & 2 & Música & $-0,78$ & 0,44 \\
\hline & & Mensagem & $-0,54$ & 0,59 \\
\hline & 3 & Música & $-1,07$ & 0,28 \\
\hline & & Mensagem & $-1,82$ & 0,07 \\
\hline \multirow{6}{*}{$\begin{array}{l}\text { FREQUÊNCIA RESPIRATÓRIA } \\
\text { (rpm) }\end{array}$} & 1 & Música & 1,07 & 0,28 \\
\hline & & Mensagem & 0,49 & 0,63 \\
\hline & 2 & Música & $-1,48$ & 0,14 \\
\hline & & Mensagem & $-1,35$ & 0,18 \\
\hline & 3 & Música & 1,26 & 0,21 \\
\hline & & Mensagem & 2,30 & 0,02 \\
\hline
\end{tabular}

Nota: Teste de Mann-Whitney: a diferença foi considerada como significativa se p inferior a 0,05 ou 0,10 e como tendência de significância se p-valor entre 0,10 e 0,20 .

Tabela 2. Valor da probabilidade de ocorrência estatística $(p)$ para a diferença da Expressão facial entre os grupos, Controle e Experimental - São Paulo, 2006.

\begin{tabular}{llll}
\hline Sessão & & Observações & $P$ \\
\hline & 1 & Música & 0,01 \\
& & Mensagem & 0,00 \\
& & Música & 0,17 \\
& Mensagem & 0,16 \\
& Música & 0,23 \\
& Mensagem & 0,34 \\
\hline
\end{tabular}

Nota: Teste Exato de Fisher: a diferença foi considerada como significativa se p inferior a 0,05 ou 0, IO e como tendência de significância se $p$ entre 0,10 e 0,20 . 
tanto durante a música Quanto durante a mensagem.

Em relação ao pulso não se encontrou alterações estatisticamente significantes, porém, analisando as médias, elas são maiores do Que a basal durante o estímulo musical e mensagem apenas na $\mathrm{I}^{\mathrm{a}}$ sessão e diminuem nas $2^{\text {a }}$ e $3^{\text {a }}$ sessões. Esses resultados são semelhantes ao estudo de caso relatado por Jones, Hux, MortonAnderson e Knepper ${ }^{(9)}$. Nesse estudo, um paciente foi submetido a 4 estímulos sonoros diferentes. Parece haver um alerta num primeiro momento, talvez na discriminação dos estímulos e, posteriormente, atenção e relaxamento frente a algo familiar, já conhecido.

Walker et $\mathrm{al}^{(10)}$, ao avaliarem o efeito da intervenção através de vozes familiares em pacientes comatosos com trauma craniano, também não encontraram variações estatisticamente significantes em relação ao pulso basal e após o estímulo mensagem.

Familiares Que vivenciam a experiência de ter um parente em estado de coma comumente relatam Que ao conversarem com ele puderam observar alterações nas mensurações de pulso dadas pelo monitor cardíaco. Essa crença também apareceu na nossa pesquisa durante a entrevista com as famílias, muitas mencionaram voluntariamente Que, Quando estavam à beira do leito do paciente e conversavam com ele, havia mudanças em sua pulsação percebidas através do monitor.

O Que podemos afirmar é Que talvez o Pulso seja uma medida fisiológica Que tenha uma grande probabilidade de variações em pacientes em estado de coma e, por isso, comparando os grupos, Experimental e Controle, não encontramos diferenças estatisticamente significantes, não sendo possível atribuir essas variações aos estímulos realizados.

Quanto à pressão arterial e saturação de $\mathrm{O}_{2}$ em pacientes comatosos frente a estímulos auditivos, o estudo de Walker, Eakes e Siebelink ${ }^{(10)}$ também não encontraram alterações estatisticamente significantes e essas variáveis também não se destacaram dentre as outras.

Para a freqüência respiratória, na sessão 3, em 9 (60\%) dos 15 pacientes do Grupo Experimental esta se tornou mais lenta durante a mensagem.

Usando a música, Aldridge et $\mathrm{al}^{(7)}$ encontraram em seu estudo uma mudança convergente a esse resultado. A respiração dos pacientes se tornava mais lenta e mais profunda durante a musicoterapia.

Jones et $\mathrm{a}^{(9)}$ relatam Que com o estímulo 'vozes familiares e de amigos', o mesmo estímulo em Que encontrou-se alterações significativas nesse estudo, a freeüência respiratória manteve-se predominantemente superior a linha basal, ou seja, se tornava mais rápida e superficial. Já frente ao estímulo musical, a freqüência respiratória apresentou pęuenas variações e sempre abaixo da linha basal.

O estudo de Walker et $\mathrm{al}^{(10)}$ também apresenta um dado interessante em relação a freQüência respiratória. Ao compararem as médias dos sinais vitais antes, durante e após uma mensagem com o objetivo de avaliar o efeito da intervenção através de vozes familiares em pacientes comatosos com trauma craniano, os autores utilizaram um nível de significância para $p$ inferior a 0,05 . Porém somente para essa variável eles encontraram um $p=0,10$ comparando a média antes e após o estímulo e um $p=0,06$ durante e após o estímulo. Mesmo os autores tendo considerado essas alterações não significativas do ponto de vista estatístico, talvez nesse momento, em Que estamos estudando algo pouco investigado e muito Questionado, fosse interessante a apresentação desses dados.
Não há dúvidas Que os sinais vitais representam um importante componente de monitorização do progresso dos pacientes durante a hospitalização, pois estes permitem a detecção alerta de recuperação atrasada ou de eventos adversos ${ }^{(17)}$. Evans et al $^{(17)}$ identificaram em uma revisão sistemática um vasto número de artigos publicados (737) Que utilizam os sinais vitais como um método de avaliação, porém, apenas uma minoria (69) apresenta resultados conclusivos e puderam ser incluídos na revisão sistemática. Os autores destacam muitas limitações em relação ao uso de medidas dos sinais vitais inclusive em relação à freQüência de mensuração e técnicas adequadas, ressaltando a necessidade de mais pesquisas com essas variáveis.

\section{A Expressão Facial}

A expressão facial dos pacientes durante cada sessão foi coletada da seguinte forma: observou-se o paciente imediatamente antes da intervenção e anotou-se sua expressão facial basal; depois disso, sua expressão facial foi gravada através de filmagem para posterior visualização.

$\mathrm{Na}$ visualização das filmagens, registraram-se pontualmente as alterações de cada paciente (movimentos de boca, de cabeça, de sobrancelha, tensão e relaxamento facial, lágrimas, abertura ocular inespecífica etc). Considerou-se uma alteração apenas expressões faciais Que não ocorreram antes da intervenção, movimentos reflexos já existentes não foram considerados, ou seja, as alterações faciais registradas foram apenas aquelas não anteriormente apresentadas pelo paciente. Porém, devido a grande diversidade de dados, não se conseguiu agrupá-los, optando então, por uma análise simplificada: ocorrência ou não de alteração da expressão facial durante a intervenção comparada à expressão basal (Tabela 2).

Para a variável expressão facial encontrou-se alterações estatisticamente significantes na sessão I tanto durante a música Quanto a mensagem. Tendências de significância foram encontradas na sessão 2 durante a música e a mensagem.

\section{CONCLUSÕES}

Foram avaliadas as seguintes variáveis: pulso, temperatura, pressão arterial sistólica, pressão arterial diastólica, saturação de $\mathrm{O}_{2}$, freqüência respiratória e expressão facial, porém encontraramse alterações estatisticamente significativas nas variáveis: saturação de $\mathrm{O}_{2}$, freeüência respiratória e expressão facial.

Para a freqüência respiratória, na sessão 3, em 9 (60\%) dos 15 pacientes do Grupo Experimental esta se tornou mais lenta e profunda durante a mensagem.

Considerando as alterações estatisticamente significantes concluiu-se Que, aparentemente, a mensagem foi um estímulo mais forte do Que a música em relação à capacidade de produzir respostas fisiológicas sugestivas de audição.

Não podemos afirmar o Quanto esses pacientes são capazes de nos ouvir, mas considerando esses resultados precisamos, no mínimo, nos preocuparmos com o Que falamos ao redor deles.

\section{CONSIDERAÇÕES FINAIS}

O momento da coleta de dados em Que se tinha um contato direto com os familiares foi uma experiência interessante. Na medida 
em Que se conversava com esse familiar esclarecendo como deveria ser a elaboração da mensagem e a escolha da música, entrava-se em contato a história desse paciente.

Durante a elaboração da mensagem observaram-se algumas coisas nos familiares: filhos Que tinham dificuldades de dizer Que amam os pais, pais Que não sabiam o gosto musical dos filhos e Que nesse momento percebiam o deslize, filhos pedindo desculpas e prometendo muitas coisas, cônjuges jurando amor eterno...

A maioria das mensagens revelou um apego a algo divino para superar as dificuldades, mesmo Que esse apego estivesse fragilizado há muito tempo. A pesquisa, para muitos familiares, parecia representar uma nova oportunidade para falar com seus entes Queridos; muitos se emocionavam e choravam.

Em relação à audição dos pacientes em estado de coma há muitas perguntas para serem feitas e muitas delas ainda não temos respostas. Por isso não se pode falar nada impensado ou inadecuado à beira do leito; é necessário tomar certos cuidados e não deixar de ser ético. A aplicabilidade desse estudo é exatamente essa.

\section{REFERÊNCIAS}

I. Houaiss A, Villar MS. Dicionário Houaiss da língua portuguesa. Rio de Janeiro: Objetiva; 200I.

2. Scotti L. Relato de alguém Que esteve em coma. [citado em 03 ago 2005]. Disponível em: http://br.geocities.com/scottibr ou http://br.geocities.com/scottibr

3. Diário de São Paulo. Italiano desperta do coma após dois anos e diz que escutava tudo. Diário de São Paulo Iperiódico on line]. 2005 out 19. [citado em 20 nov 2005]. Disponível em: http://www.diariosp.com.br/mundo/default.asp?Editoria $=38 \& \mathrm{id}=359449$.

4. Lent R. Cem bilhões de neurônios: conceitos fundamentais de neurociência. São Paulo: Atheneu; 2005.

5. Smeltzer SC, Bare BG. Tratado de enfermagem médicocirúrgica. Rio de Janeiro: Guanabara Koogan, 2002.

6. Porto CC. Semiologia médica. Rio de Janeiro: Guanabara Koogan 2005.

7. Aldridge D, Gustorff D, Hannich HJ. Where am I? Music therapy applied to coma patients. I R Soc Med 1990; 83(6): 345-6.

8. Sisson R. Effects of auditory stimuli on comatose patients with head injury. Heart Lung 1990; 19(4): 373-8.

9. Jones R, Hux K, Morton-Anderson A, Knepper L. Auditory stimulation effect on a comatose survivor of traumatic brain injury. Arch Phys Med Rehabil 1994; 75: 164-71.
10. Walker IS, Eakes GG, Siebelink E. The effects of familial voice interventions on comatose head-injured patients. I Trauma Nurs 1998;5(2):41-6.

II. Ferreira MIPR. A comunicação entre a equipe de saúde e o paciente em coma: dois mundos diferentes de interação [tese]. Florianópolis: Universidade Federal de Santa Catarina; 2000.

12. Puggina ACG, Silva MIP da, Gatti MFZ, Graziano KU, Kimura M. A percepção auditiva nos pacientes em estado de coma: uma revisão bibliográfica. Acta Paul Enferm 2005; I 8(3):3 13-9.

13. Fletcher RH, Fletcher SW, Wagner EH. Epidemiologia clínica: elementos essenciais. $3^{\mathrm{a}}$ ed. Porto Alegre: Artmed; 1996.

14. Pierin AMG, Padilha KG, Cruz DALM. Caracterização dos pacientes de duas unidades de terapia intensiva (UTI): condições bio-sociais, processo de internação e intervenções terapêuticas. Rev Esc Enferm USP 1990; 24(3): 371-88.

15. McCaffrey R, Locsin RC. Music listening as a nursing intervention: a symphony of practice. Holist Nurs Pract 2002; 16(3): 70-7.

16. Nascimento AR, Caetano IA. Pacientes de UTI: perspectivas e sentimentos revelados. Rev Nursing 2003; 6(57): 12-7.

17. Evans D, Hodgkinson B, Berry J. Vital signs in hospital patients: a systematic review. Int I Nurs Stud 2001;38:643-50. 